\section{DEVELOPMENT OF MULTI FREQUENCY ELECTRICAL IMPEDANCE TOMOGRAPHY FOR RECTANGULAR GEOMETRY BY FINITE VOLUME METHODS}

Khusnul Aina, Deddy Kurniadib, Mokhamad Fakhrul Ulumc, Lina Choridahd, Utriweni Mukhayyare, Agah D. Garnadif, Nurhuda Hendra Setyawand, Bayu Ariwantog

aBiomedical Engineering, Airlangga University, Surabaya, Indonesia bPhysics Engineering, Institute of Technology Bandung, Bandung, Indonesia

cVeteranary Clinic Reproduction and Pathology, Institute of Agriculture Bogor, Bogor, Indonesia

aedicine, Gadjah Mada University, Yogyakarta, Indonesia

eStatistics, Institute of Technology Bandung, Bandung, Indonesia

fMathematics and Sciences, Institute of Agriculture Bogor, Bogor, Indonesia

gPhysics, Airlangga University, Surabaya, Indoensia
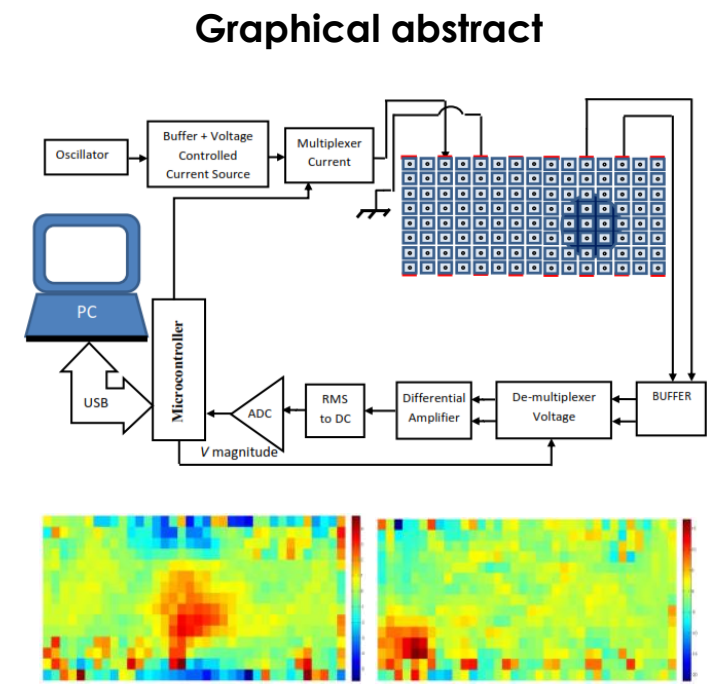

\begin{abstract}
Electrical impedance tomography (EIT) is a non-invasive technique for imaging the electrical conductivity and permittivity in the body by measuring the electrical potential on the surface. One of the EIT applications in the medical field is breast cancer detection. The study proposed a rectangular geometry EIT multi-frequency device based on the Finite Volume Method (FVM) reconstruction method. This device can be used to complement mammography images into dual EIT-mammography modalities. The EIT device built of DDS AD9850 as a programmable multifrequency sinusoidal generator, AD620 as instrument amplifier, AD536 as an AC converter, and ADS1115 as an ADC 16 bit. These devices can scan at the maximum frequency of $100 \mathrm{kHz}$. The device has been tested with a phantom of distilled water and carrots in the center and on the sides. The reconstruction methods one step different frequency produces a rectangular geometric image with anomalies in the depth and at the surface clearly.

Keywords: Multi frequency, electrical impedance tomography, finite volume methods, rectangular geometry, breast cancer
\end{abstract}

(c) 2022 Penerbit UTM Press. All rights reserved 


\subsection{INTRODUCTION}

Electrical impedance tomography (EIT) provide low cost, real-time, non-invasive imaging modality that can image the electrical conductivity and permittivity of the body [1]. The electrodes are attached to the surface of the body and a certain current pattern is introduced into them through the electrodes. The distribution of the electrical conductivity and permittivity in the body is reconstructed based on the measurement of the electric potential data of the electrodes positioned around the boundary $[2,3]$.

EIT was applied in the medical field, including the monitoring of intracranial hemorrhages or hematomas [4], cancer detection were lung cancer [5], skin cancer [6] and breast cancer [7], study of pelvic fluid accumulation [8], pulmonary ventilation analysis [9], blood pressure measurement [10], lung imaging [11], Cardio-Pulmonary Monitoring [12], Hyperthermia Treatment [13], Pediatric Lung Disease [14], Breast Imaging [15], Brain Imaging [16].

Several researchers developed EIT devices including a telemedicine system based on the fast and portable EIT [17]. Hartinger et.al. developed EIT based system with a handheld probe comprising 16 disposable semi-invasive electrodes for the early diagnosis of skin cancer [18], and a multifrequency EIT system for biomedical imaging [19]. The variety and importance of the EIT for biomedicine, that it is necessary to develop EIT, especially in the biomedical field.

In general, the design of the object surface boundaries is circular because it represents many objects including the thorax, head, abdomen, and breasts. In certain conditions, it used box geometry such as mammography. The research used mammography geometry has been carried out by modeling a rectangular geometry with an arrangement of electrodes at the top and bottom [20].

The success of mammography geometry is then used as a dual-modality of mammography and EIT [21], and thermography and EIT [22]. To support this research, a simulation study of $2 \mathrm{D}$ rectangular geometry finite volume methods (FVM) has been conducted by K. Ain et al. with good results [-23]. FVM has several advantages than Finite Element Methods (FEM). Several researchers have reported many advantages of FVM than FEM [24] including FVM and FEM methods are qualitatively similar but different quantitatively from theoretical solutions. In theory, FVM provides a better approach than FEM [25]. FVM can produce a higher mesh density than FEM [26]. Computationally, FEM takes longer computation time and requires more memory than FVM [26]. Based on this background, our study is to realize a $2 \mathrm{D}$ rectangular geometry multi-frequency EIT device using the FVM method.

\subsection{METHODOLOGY}

This research focuses on the multi-frequency electrical impedance tomography system for planar objects. The research started by designing the device then testing the performance of EIT data acquisition system modules. The modules include: signal generator, DC block, Voltage Converter Current Source (VCCS), and voltage measurement system shown in Figure 1.

We used the signal generator module DDS AD9850 that could produce a sinus signal offset DC with frequencies from 0 to $40 \mathrm{MHz}$ [27]. The DC block or a HPF (High Pass Filter) was used to shift the DC sinus signal into an AC sinus signal. The VCCS provides constant current that was needed as the current injection. The current frequency could be changed by adjusting the signal generator frequency. The target of current injection in the EIT system was adjusted using a multiplexer. Meanwhile, the target of voltage measurement was adjusted using a demultiplexer. The voltage between electrodes was measured using the AD536 instrument amplifier that converted $A C$ signals into $D C$ signals so that the results could be read by the ADC.

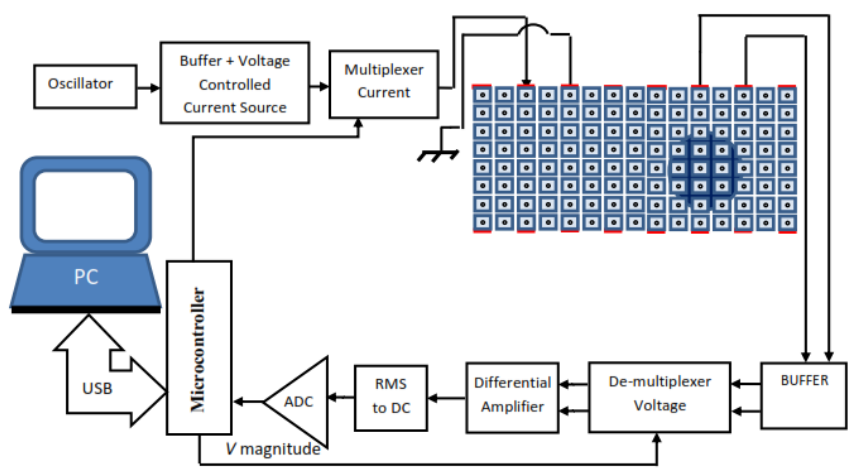

Figure 1 Multi-frequency electrical impedance tomography system for rectangular objects

The modules were integrated and synchronized into an EIT system. Data acquisition was an experimental step to test the performance of the $2 \mathrm{D}$ multi-frequency EIT system. The hardware was tested by doing a scan to a breast phantom, which produced experimental electric potential data. The phantom water and carrot as a substitute for breast and cancer, based on electrical impedance spectroscopy data that carrot in water is similar to cancer in the breast $[28,29]$. Reconstruction was done using the relative reconstruction method from FVM forward problem [18], which makes the multifrequency electrical impedance tomography device suitable for breast cancer detection. Relative method assumes that the potential change boundary is a linear function of the change in conductivity [30,31]. The relative reconstruction methods can be written as $[\delta \sigma]=([S] T[S]+\alpha I)-1[S] T[\delta V]$. Where $[\delta \mathrm{V}]$ is change of boundary potential, $[\mathrm{S}]$ is sensitivity matrix, 
$\alpha$ is regularisation parameter, $I$ is identity matrix and $[\delta \sigma]$ is relative conductivity. The analysis was done by comparing the object phantom with the reconstructed image obtained.

\subsection{RESULT AND DISCUSSION}

Results obtained in this research include Adjustment of signal frequency, DC block or HPF (High Pass Filter), VCCS, voltage measurement, and multi-frequency EIT system for planar objects.

(1) Adjustment of signal frequency

AD9850 is a controllable generator which can generate signals DC offset at frequencies $0 \mathrm{~Hz}$ to $40 \mathrm{MHz}$. ATMEL designed an AD9850 DDS open source in the module that supports the Arduino. The execution of the program produced DC offset analog signals from frequency 0 until $1 \mathrm{MHz}$ is shown in Figure 2, more than $1 \mathrm{MHz}$, it will produce a bad sine wave signal.

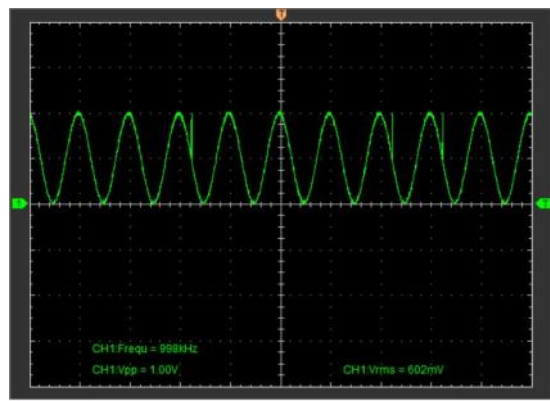

Figure 2 Sinus signal produced by DDS module AD9850 at frequencies $1 \mathrm{MHz}$

Figure 2 shows that the signals produced have a nice form, however, frequencies over $40 \mathrm{MHz}$ showed an unstable signal. The Vrms produced were plotted versus frequency, shown in Figure 3. The graph shows that the Vrms produced was $600 \mathrm{mV}$ stable at frequencies up to $1 \mathrm{MHz}$ shown in Figure 3.

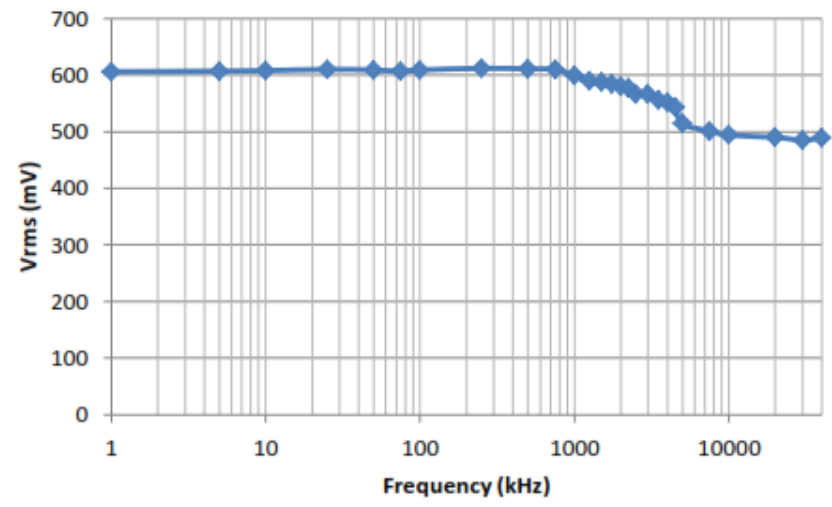

Figure $3 \mathrm{~V}_{\mathrm{rms}}$ voltage produced by DDS module at frequencies up to $40 \mathrm{MHz}$.
(2) Block DC

In order for the DC signal to become an AC signal, the signal must be passed through a DC block, as shown in Figure 4. The mechanism of the block DC shifts down or changes the DC off set into zero since the DC off set voltage is filtered by the DC block. This circuit is exactly the same as a high pass filter, using $C$ $=100 \mu \mathrm{F}$ and $\mathrm{R}=1 \mathrm{k} \Omega$, theoretically will produce HPF with a cutoff of $1.6 \mathrm{~Hz}$.

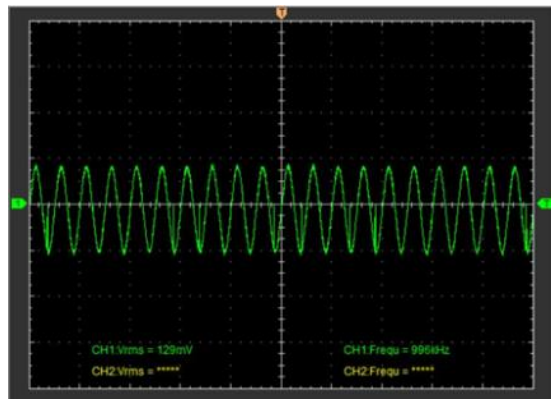

Figure $4 \mathrm{AC}$ sinus signals produced from the $\mathrm{DC}$ block at frequencies $1 \mathrm{MHz}$

The consequence of the DC block circuit is the disappearance of signals with low frequencies as shown in Figure 5. When the voltage produced is plotted with respect to frequency, it results in low Vrms at frequencies less than $10 \mathrm{~Hz}$.

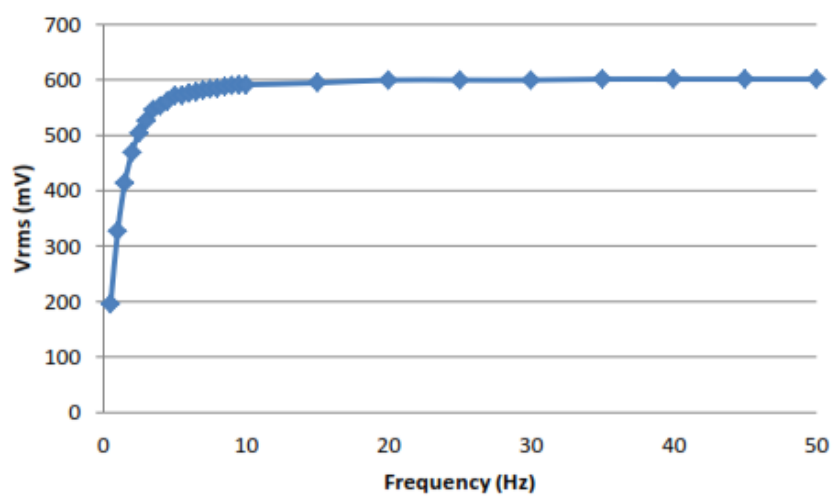

Figure $\mathbf{5} \mathrm{V}_{\mathrm{rms}}$ produced after through pass DC block

\section{(3) VCCS (voltage controlled current source)}

The VCCS schematic shown in Figure 6 consists of a high pass filter, multilevel amplifier, buffer. The research uses VCCS dual op-Amp with $R_{F}, R_{s}$, and $R_{1}$ are $2 \mathrm{k} \Omega$ shown in Figure 6. The VCCS characterization related to current stability against frequency and load is shown in Figure 7. This VCCS can produces a constant electric current of $1 \mathrm{~mA}$ up to a frequency of $150 \mathrm{kHz}$ with a load of $1 \mathrm{k} \Omega, 100 \mathrm{kHz}$ with a load of $2 \mathrm{k} \Omega$, and $80 \mathrm{kHz}$ with a load of $3 \mathrm{k} \Omega$. 


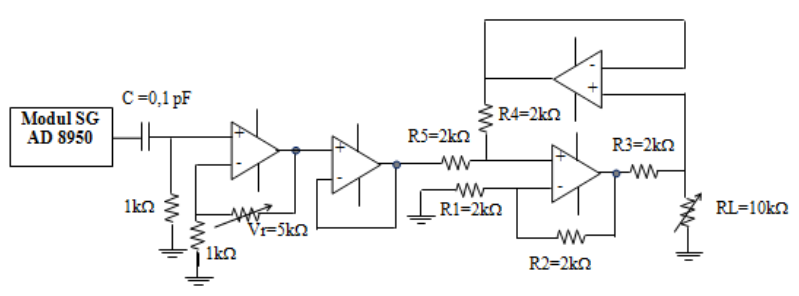

Figure 6 Schematic of VCCS

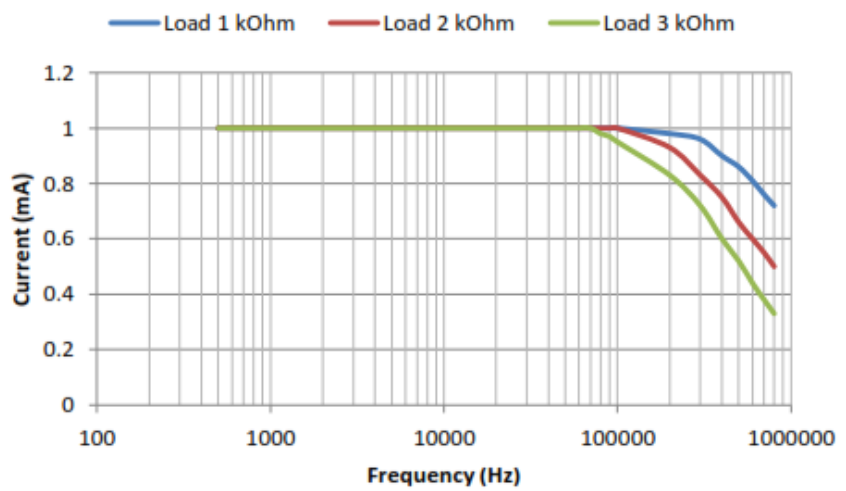

Figure 7 The curve of VCCS current stability for the load at a frequency of 10 to $100 \mathrm{kHz}$

\section{(4) Voltage Measurement}

Voltage measurement composed of the buffer, instrument amplifier, voltage converter, and ADC showed in Figure 8. The buffer used to maintain the voltage measured, instrumentation amplifier used to measure the potential between electrodes, a voltage converter used to convert VAC into VRMS, and ADC used to convert analog to digital data. It needs 16 buffers to keep the potential measured from 16 electrodes.

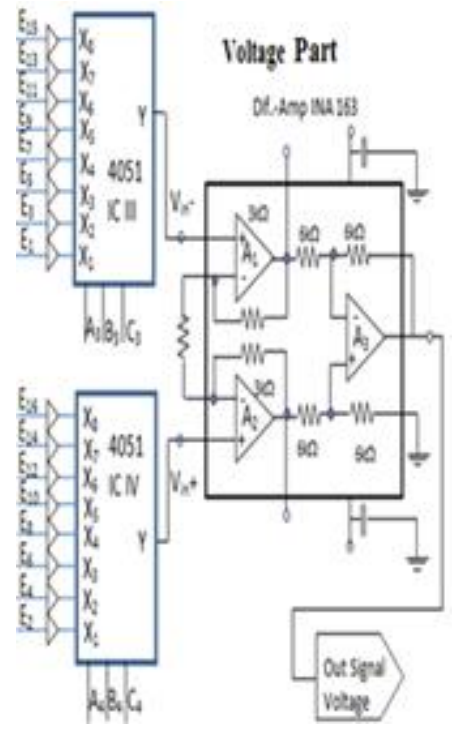

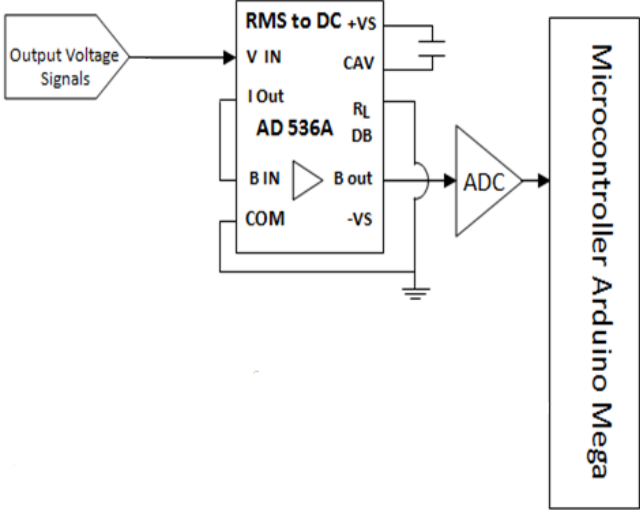

Figure $\mathbf{8}$ Voltage Measurement system

The output of the function generator was connected to each input port of the buffer and the output of the buffer was connected to the digital oscilloscope to observe until the $500 \mathrm{kHz}$ waveform Rms voltage. The waveform can be seen in Figure 9, in which green line is input signal and yellow line is output signal.

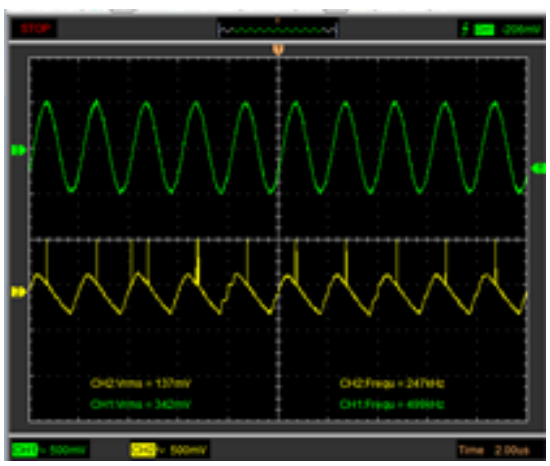

Figure 9 Input and Output Passed through Buffer at Frequencies of $500 \mathrm{kHz}$

The output waveform of the buffer was no sinusoidal at the frequency of $500 \mathrm{kHz}$ or above, however, it was triangular. The performance revealed that the frequency which could be inputted to the buffer between $10 \mathrm{~Hz}$ to $100 \mathrm{kHz}$.

Testing was conducted by injecting sinusoidal signals with varied frequencies and the input voltage and then measuring the output RMS voltage of the AD620. The input-output signals and RMS are shown in Figure 10 and Figure 11 respectively, in which the green line is input and the yellow line is output. The performance revealed that the frequency which could be inputted to the instrument amplifier between $10 \mathrm{~Hz}$ to $100 \mathrm{kHz}$. 


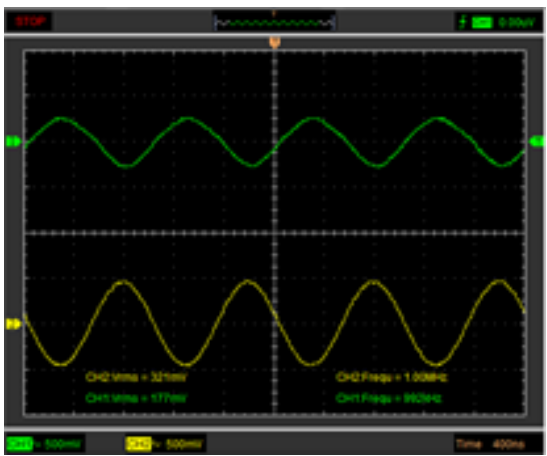

Figure 10 Comparation between input and output signal of AD620 at frequencies of (a) $10 \mathrm{kHz}$ (b) $50 \mathrm{kHz}$ (c) $100 \mathrm{kHz}$ (d) $1 \mathrm{MHz}$

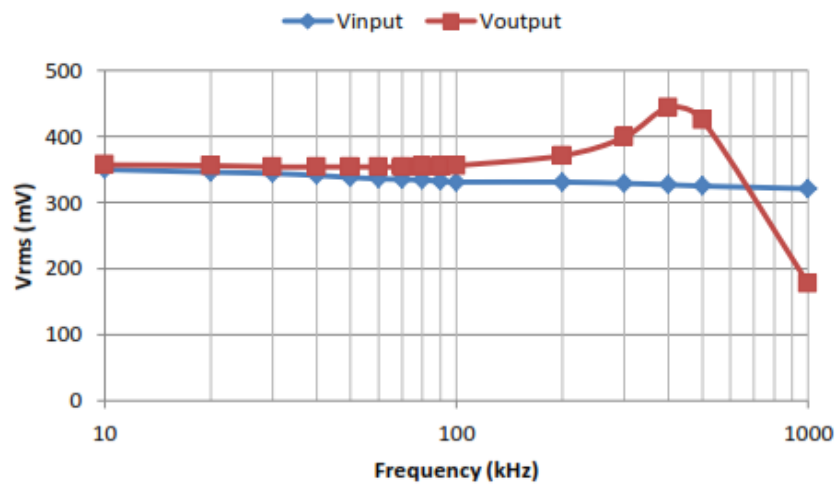

Figure 11 Performance of AD620 at varied frequencies and input voltage

The performance of AD536 is shown in Figure 12 which points out that at frequencies $500 \mathrm{kHz}$, the Vdc obtained was stable. The yellow line is the input signal and the green line is Vdc produced as output.

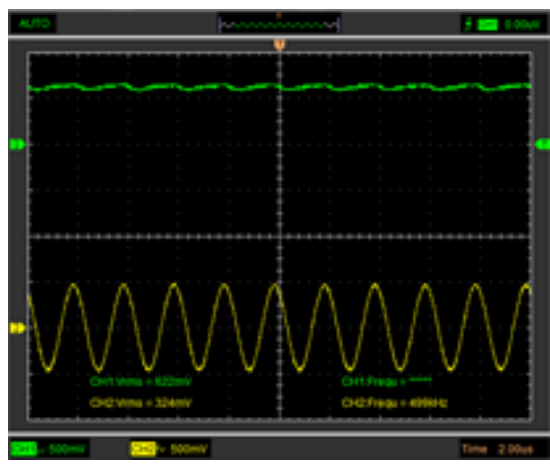

Figure 12 Sinusoidal Waveform of the Input and RMS Output of AD536 at frequencies of $500 \mathrm{kHz}$

The Microcontroller is equipped with a 10 bit ADC, however, to increase read a data, we used an ADS 1115 as 16 bits ADC. The test of ADC was conducted by adding a variable resistor so that the voltage read by the ADC changed. The ADC testing with respect to the input voltage shown in Figure 13. The results have $R^{2}=1$, means that it is linear.

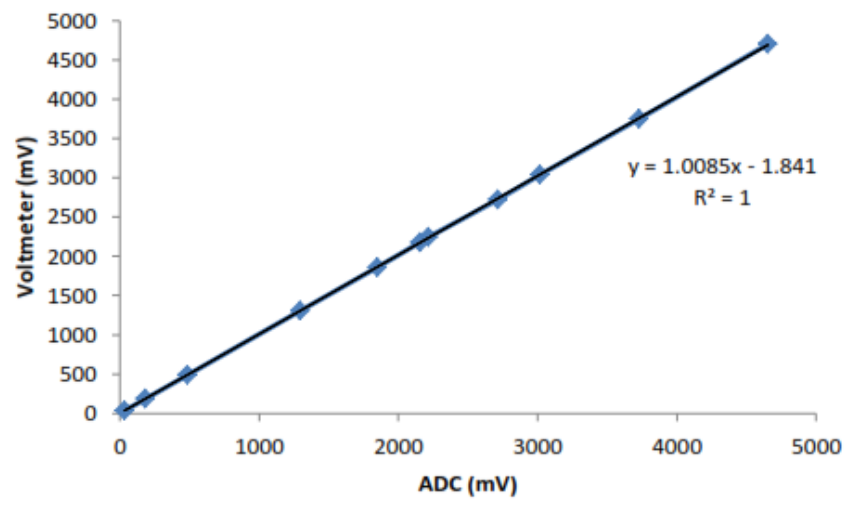

Figure 13 Testing Results of 16 bit ADC

(5) Multi-frequency ElT System for Planar Objects

The multi-frequency EIT device was composed of the module components that were already tested and analyzed. All of the modules were arranged into a multi-frequency EIT circuit shown in Figure 14.

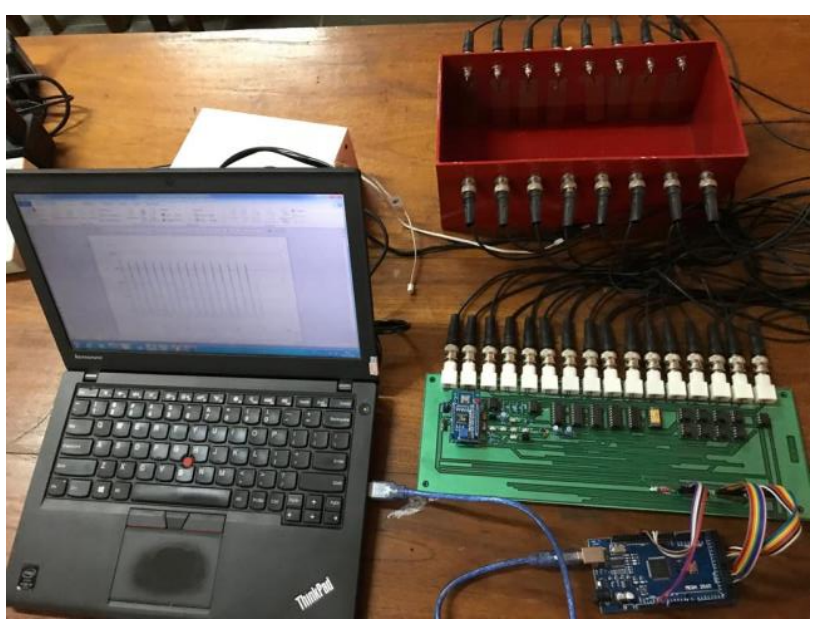

Figure 14 Multi-frequency EIT for planar objects

The EIT system was used to scan the experimental objects which were in the middle and edge posistion shown in Figure 15. The two frequencies used in the scanning process were $10 \mathrm{kHz}$ and $100 \mathrm{kHz}$. The determination of the two selected frequencies is based on the electrical impedance spectroscopy data, namely the two frequencies that have the largest impedance difference so that produce the maximum image contrast. Potential data was obtained and the results are shown in Figure 16. 


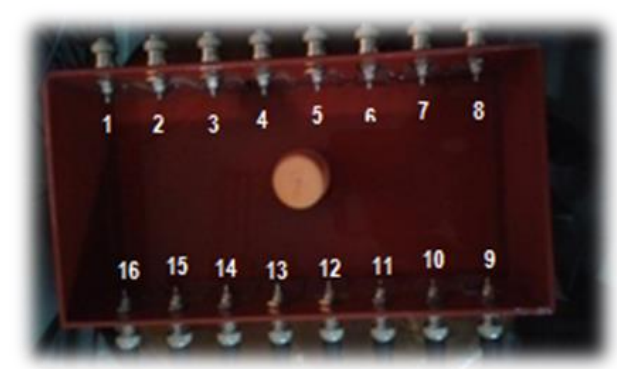

(a)

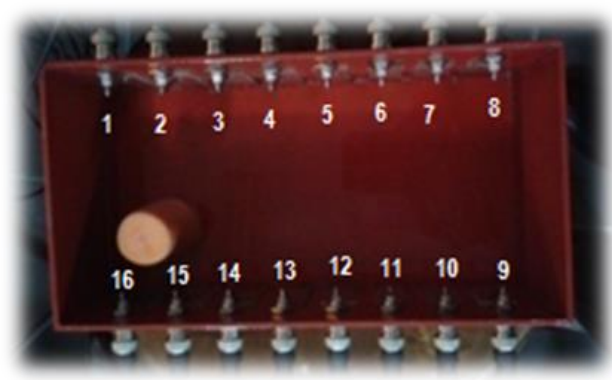

(b)

Figure 15 Experimental phantom that consists of distilled water and a carrot, both to represent normal and anomaly breasts with (a) anomaly in the middle (b) anomaly at the edge

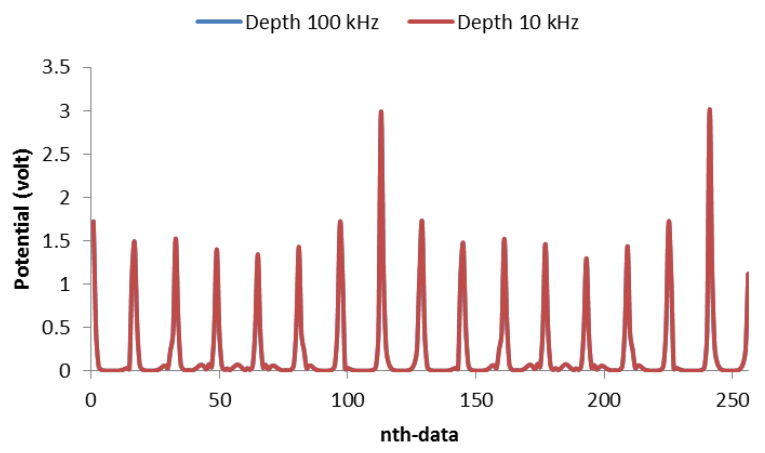

(a)

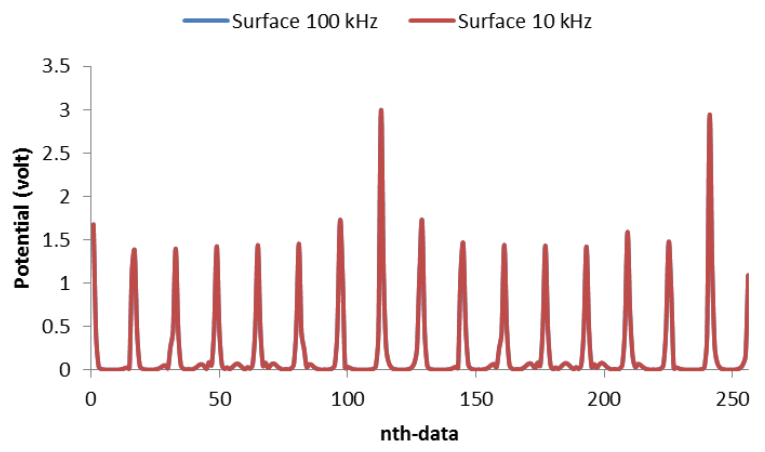

(b)

Figure 16 The experimental phantom scanning data of 16 electrodes using neighboring data collection methode at frequencies of $10 \mathrm{kHz}$ and $100 \mathrm{kHz}$ with anomalies (a) in the middle (b) at the edge
The potential data of the experimental phantom object in Figure 16 was then reconstructed using back projection with $\alpha=5 \times 10^{-8}$ and $\alpha=5 \times 10^{-9}$ using data at frequencies of $100 \mathrm{kHz}$ and $10 \mathrm{kHz}$ as reference. The reconstructed images were shown in Figure 17.

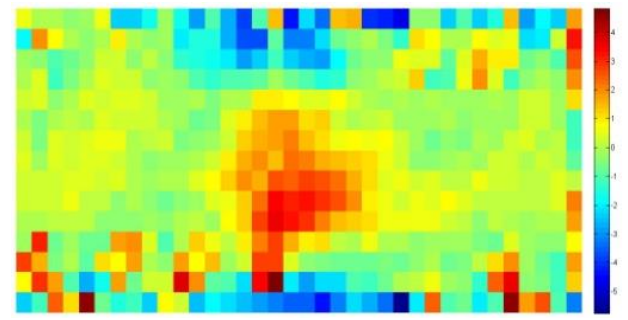

(a)

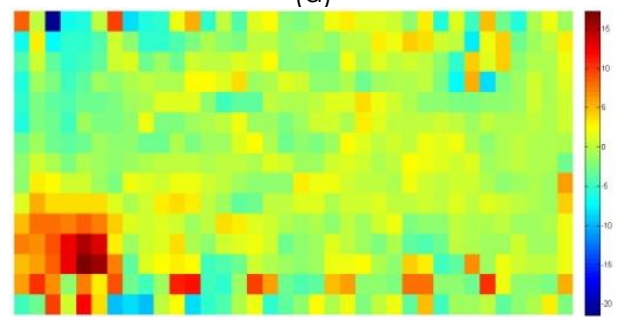

(b)

Figure 17 Reconstructed images using back projection with data $V_{f}=100 \mathrm{kHz}$ and $V_{\text {ref }}=10 \mathrm{kHz}$ using neighboring data collection method (a) anomaly in the middle (b) anomaly at the edge.

Figure 17 shown that the experiment successfully reconstructed the image of a phantom both inside and surface positions. Therefore, it can be said that the EIT device has the ability to produce reconstructed images from the impedance of the experimental object.

Each rectangular geometry EIT multi-frequency modul has been properly confirmed at a maximum frequency of $100 \mathrm{kHz}$. This study is a follow-up to previous studies, it has studied the forward problem of the FVM method with rectangular geometry [23]. Rectangular geometry is very suitable using the FVM method so that the reconstruction process is faster and more memory efficient than FEM. The rectangular geometry fits mammography so well that it is possible to become dual modality EIT and mammography [21]. The researcher has done it with 3D but only uses a number of electrodes on the top 16 and bottom 16. The system can generates 8 slices with each slice using 8 electrodes to produce low resolution images. In our research, although it is still $2 D$, each slice uses 16 electrodes so that the resulting image has a higher resolution.

\subsection{CONCLUSION}

The development of the multi-frequency EIT rectangular geometry device has been carried out by utilizing the DDS AD9850 as a programmable multifrequency sinusoidal generator, the AD620 instrument 
amplifier as a linear amplifier, the AD536 as an AC converter, and the ADS 1115 as a 16 bit ADC. Each component has been tested for its ability in the frequency range up to $100 \mathrm{kHz}$. The EIT system was tested using a phantom consisting of distilled water and carrots. Reconstruction using the linear method of the FVM forward problem with a frequency difference of $10 \mathrm{kHz}$ and $100 \mathrm{kHz}$ produced a reconstruction image of an anomaly in the center or near the surface position successfully.

\section{Acknowledgment}

The authors would like to express gratefulness to the Indonesian Ministry of Research and high education by Institution of Research and Innovation for funding this research through the RKI Project No. 250/UN3.14/PT/2020.

\section{References}

[1] Wu, Y., Hanzaee, F. F., Jiang, D., Bayford, R. H. Demosthenous, A. 2021. Electrical Impedance Tomography for Biomedical Applications: Circuits and Systems Review. IEEE Open Journal of Circuits and Systems. DOI: 10.1109/OJCAS.2021.3075302.

[2] Kao, T. J., Saulnier, G. J., Xia, H., Tamma, C., Newell, J. C. and Isaacson, D. 2007. A Compensated Radiolucent Electrode Array for Combined EIT and Mammography. Physiological Measurement. 28(7): S291-S299.

DOI: //dx.doi.org/10.1088/0967-3334/28/7/S22.

[3] Harikumar, R., Prabu, R., Raghavan, S. 2013. Electrical Impedance Tomography (EIT) and Its Medical Applications. International Journal of Soft Computing and Engineering (IJSCE). 3(4).

[4] Ayati, S. B., Bouazza-Marouf, K., and Kerr, D. 2015. In Vitro Localisation of Intracranial Haematoma using Electrical Impedance Tomography Semi-array. Medical Engineering \& Physics. 37(1): 34-41.

DOI: 10.1016/j.medengphy.2014.10.001

[5] Gao, J., Yue, S., Chen, J., and Wang, H. 2014 Classification of Normal and Cancerous Lung Tissues by Electrical Impendence Tomography. Bio-medical Materials and Engineering. 24(6): 2229-2241.

DOI: 10.3233/BME-141035.

[6] Moqadam, S. M., Grewal, P. K., Haeri, Z., Ingledew, P. A. Kohli, K. and Golnaraghi, F. 2018. Cancer Detection based on Electrical Impedance Spectroscopy: A Clinical Study. Journal of Electrical Bioimpedance. 9: 17-23.

[7] Xu, F., Li, M., Li, J., Jiang, H. 2021. Diagnostic Accuracy and Prognostic Value of Three-dimensional Electrical Impedance Tomography Imaging in Patients with Breast Cancer. Gland Surgery. 10(9): 2673-2685. DOI: 10.21037/gs-21-348.

[8] Li, R., Gao, J., Li, Y., Wu, J., Zhao, Z., and Liu, Y. 2016. Preliminary Study of Assessing Bladder Urinary Volume using Electrical Impedance Tomography. Journal of Medical and Biological Engineering. 36(1): 71-79. DOI: https://doi.org/10.1007/s40846-016-0108-1.

[9] Bordes, J., Goutorbe, P., Cungi, P. J., Boghossian, M. C. and Kaiser, E. 2016. Noninvasive Ventilation during Spontaneous Breathing Anesthesia: An Observational Study using Electrical Impedance Tomography. Journal of Clinical Anesthesia. 34: 420-426. DOI: https://doi.org/10.1016/j.jclinane.2016.04.016.

[10] Proenca, M., Braun, F., Sola, J., Thiran, J. P., and Lemay, M. 2017. Noninvasive Pulmonary Artery Pressure Monitoring by
EIT: a Model-based Feasibility Study. Medical \& Biological Engineering \& Computing. 55: 949-963.

DOl: https://doi.org/10.1007/s11517-016-1570-1.

[11] Adler, A., Berthiaume, Y., Guardo, R., and Amyot, R. 1995. Imaging of Pulmonary Edema with Electrical Impedance Tomography. Proceedings of 17th International Conference of the Engineering in Medicine and Biology Society. 557-558.

DOI: 10.1 109/IEMBS.1995.575248.

[12] Putensen, C., Hentze, B., Muenster, S., and Muders, T. 2019. Electrical Impedance Tomography for Cardio-Pulmonary Monitoring. Journal of Clinical Medicine. 8(8): 1176. DOI: org/10.3390/jcm8081176.

[13] Conway, J. 1987. Electrical Impedance Tomography for Thermal Monitoring of Hyperthermia Treatment: An Assessment using in Vitro and In Vivo Measurements. Clin. Phys, Physiol. Meas. 8(A): 141-146. DOI: $10.1088 / 0143-0815 / 8 / 4 a / 018$.

[14] Pham, T. M. T., Yuill, M., Dakin, C., and Schibler, A. 2011. Regional Ventilation Distribution in the First 6 Months of Life. European Respiratory Journal (ERJ). 1 (37): 4 919-924. DOI: $10.1183 / 09031936.00034310$

[15] Zain N. M., and Chelliah K. K. 2014. Breast imaging using electrical impedance tomography: correlation of quantitative assessment with visual interpretation. Asian Pacific Journal of Cancer Prevention. 15(3): 1327-1331. DOI: 10.7314/apjcp.2014.15.3.1327.

[16] Holder, D., S., Rao, A., and Hanquan, Y. 1996. Imaging of Physiologically Evoked Responses by Electrical Impedance Tomography with Cortical Electrodes in the Anaesthetized Rabbit. Physiol. Meas. 17: A179-A186. DOI: 10.1088/0967-3334/17/4a/022.

[17] Zhou, Z., Nan, L., Hui, X., Jin, G., Zhaolin, S., Haijun, L., Hongqi, Y. 2012. The Design and Implementation of a Portable EIT Telemedicine System. International Conference on Intelligent System Design and Engineering Application, IEEE. 571-575.

DOI: $10.1109 /$ ISdea.2012.741.

[18] Hartinger, A. E., and Gagnon, H. 2012. EIT System and Reconstruction algorithm adapted for Skin Cancer Imaging. The 11th International Conference on Information Sciences, Signal Processing and their Applications. 798-803.

DOI: 10.1109/ISSPA.2012.6310662.

[19] Bera, T. K., and Nagaraju, J. 2012. Multifrequency Electrical Impedance Tomography (EIT) System for Biomedical Imaging, IEEE. DOI: $10.1109 /$ SPCOM.2012.6290216.

[20] Choi, M. H., Kao, T. J., Isaacson, D., Saulnier, G. J., and Newell, J. C. 2007. A Reconstruction Algorithm for Breast Cancer Imaging With Electrical Impedance Tomography in Mammography Geometry. IEEE Transactions on Biomedical Engineering. 54(4). DOI: $10.1109 /$ TBME.2006.890139.

[21] Kao, T. J., Saulnier, G. J., Xia, H., Tamma, C., Newell, J. C. and Isaacson, D. 2007. A Compensated Radiolucent Electrode Array for Combined EIT and Mammography. Physiological Measurement. 28: S291-S299. DOI: 10.1088/0967-3334/28/7/S22.

[22] J. Zuluaga-Gomez, N. Zerhouni, Z. Al Masry, C. Devalland \& C. Varnier. 2019. A Survey of Breast Cancer Screening Techniques: Thermography and Electrical Impedance Tomography. Journal of Medical Engineering \& Technology. 1-16. DOI: 10.1080/03091902.2019.1664672.

[23] Ain, K., Wibowo, R. A., Soelistionot, S. 2017. Modeling of Electrical Impedance Tomography to Detect Breast Cancer by Finite Volume Methods. Journal of Physics: Conf. Series. 853: 012001.

[24] O'Callaghan, S., Walsh, M., McGloughlin, T. 2003. Comparison of Finite Volume, Finite Element and Theoretical Predictions of Blood Flow through an Idealised Femoral Artery. Summer Bioengineering Conference. Sonesta Beach Resort in Key Biscayne, Florida. 
[25] Fallah, N. A., Bailey, C., Cross, M., and Taylor, G. A. 2000. Comparison of Finite Element and Finite Volume Methods Application in Geometrically Nonlinear Stress Analysis. Applied Mathematical Modelling. 24: 439-455. DOl: https://doi.org/10.1016/\$0307-904X(99)00047-5.

[26] Molina-Aiz, F. D., Fatnassi, A, H., Boulard, T., Roy, J. C., and Valera, D. L. 2010. Comparison of finite Element and finite Volume Methods for Simulation of Natural Ventilation in Greenhouses. Computers and Electronics in Agriculture. 72: 69-86.

DOI: https://doi.org/10.1016/j.compag.2010.03.002.

[27] Arisandi, E. D. 2015. Design and Development of Low Cost Flight Termination System Encoder based on Dds Ad9850 for Flight Vehicle. Proceedings of International Seminar of Aerospace Science and Technology III. 52-58.

[28] Amin, N., Rayhan, S., Anik, A. A., Jameel, R. 2016. Modelling and Characterization of Cell Abnormality using Electrical Impedance Spectroscopy (EIS) System for the
Preliminary Analysis to Predict Breast Cancer. Proceedings of International Conference on Research Intellegence and Communacation.

[29] Packham, B., Koo, H., Romsauerova, A., Ahn, S., Mcewan, A., Jun, S. C., and Holder, D. S. 2012. Comparison of Frequency Difference Reconstruction Algorithms for the Detection of Acute Stroke using EIT in a Realistic Headshaped Tank. Physiol. Meas. 33: 767-786.

[30] Borsic, A. 2002. Regularisation Methods for Imaging from Electrical Measurements. A Thesis of Philosophy Doctor. Oxford Brookes University.

[31] Chen, X. Y., Wang, H. X., Newell, J. 2011. Lung Ventilation Reconstruction by Electrical Impedance Tomography Based on Physical Information. 3rd International Conference on Measuring Technology and Mechatronics Automation, IEEE Computer Society. 